\title{
Model Tags: Direct 3D Tracking of Heart Wall Motion from Tagged MR Images
}

\author{
Alistair A. Young \\ Department of Anatomy with Radiology, \\ University of Auckland, Private Bag 92019, Auckland, New Zealand. \\ a. young@auckland.ac.nz, http://ww.auckland.ac.nz/anat/ayoung/
}

\begin{abstract}
A method is presented for the reconstruction of 3D heart wall motion directly from tagged magnetic resonance (MR) images, without prior identification of ventricular boundaries or tag stripe locations. Model tags were created as material surfaces which defined the location of the magnetic tags within the model. Image-derived forces acted on the model tags, while the model could also be manipulated by a small number of user-controlled guide points. The method was applied to simulated images in which the true motion was specified, as well as to clinical images of a normal volunteer. The RMS errors in displacement and strain calculated from the simulated images were similar to those obtained using previous stripe tracking and model fitting methods. A significant improvement in analysis time was obtained for the normal volunteer, making the method more clinically viable.
\end{abstract}

\section{Introduction}

Magnetic resonance (MR) tissue tagging is a useful clinical tool for the noninvasive measurement of heart wall motion [1], [2]. Typically, multiple parallel tagging planes of magnetic saturation are created orthogonal to the image plane in a short time interval $(5-12 \mathrm{msec})$ on detection of the $\mathrm{R}$ wave of the ECG. Often a grid of tag planes is created, whose intersection with the image plane give rise to dark bands ("image stripes"), 1-2 $\mathrm{mm}$ in width and spaced 5-10 $\mathrm{mm}$ apart, which deform with the tissue and fade according to the time constant T1 ( $700 \mathrm{msec}$ in myocardium). Techniques for stripe tracking and strain estimation have been developed and validated in both $2 \mathrm{D}[3]$ and 3D [4]. Recently, a number of clinical studies have used MR tagging to characterize regional left ventricular wall motion and deformation in normal and diseased hearts [5],[6]. The clinical utility of this technique is currently limited by the prohibitively long time required for image analysis. Most analysis methods require the prior extraction of the inner and outer boundaries of the left ventricle in each image together with the localization of the image tag stripes in each frame [4],[7],[8],[9],[10],[11],[12]. Several semi-automatic methods have been developed for tracking the tags and identifying the boundaries [4],[13],[14]. However, the image intensity information is insufficient to completely characterize the boundary and tag locations, due to limited spatial and temporal resolution, lack of contrast between muscle and 
blood, and respiration and gating artifacts. User interaction with the tracking and segmentation processes is therefore essential. As 3D studies typically comprise more than 200 images (5-12 short axis slices and 5-8 long axis slices, each with 5-20 frames), the time required for user interaction can be substantial. This paper describes a method for reconstructing regional left ventricular (LV) motion and deformation directly and simultaneously from a set of long and short axis tagged MR images, without the need for separate boundary and image stripe tracking.

\section{Methods}

\subsection{Finite Element Model}

As done previousiy, a 16 element finite element (FE) model was constructed to describe the geometry and motion of the left ventricle [4],[6]. Each element employed bicubic Hermite interpolation in the circumferential and longitudinal directions, with linear interpolation transmurally. Nodes shared position and derivative information between elements, giving $C^{1}$ continuity. Within each element, the geometric field $\mathbf{x}$ was given as a function of element (material) coordinates $\xi$ as a weighted average of nodal values:

$$
\mathbf{x}\left(\xi_{1}, \xi_{2}, \xi_{3}\right)=\sum_{n} \Psi_{n}\left(\xi_{1}, \xi_{2}, \xi_{3}\right) \mathbf{x}^{n}
$$

where $\mathbf{x}^{n}$ are the nodal values and $\Psi_{n}$ are the element basis functions. Note that the element coordinates $\xi$ of material points do not change (by definition) as the model deforms.

As a first step in the motion reconstruction problem, the approximate geometry of the LV at end-diastole (ED) must be determined. This was done interactively by fitting a small number of guide points by linear least squares. A smoothing term constrained the model to smoothly approximate the guide points. In practice, the geometry of a subsequent time frame (eg. the second or third frame) was constructed first, then deformed to ED using the relative motion of the tag stripes to indicate the displacement of the guide points.

\subsection{Model Tags}

Image stripes provide information about the underlying deformation of the heart but do not represent the motion of material points. Due to the tomographic nature of the imaging process, material points move through the fixed image slice planes during the deformation. This can result in the appearance and disappearance of image stripes due to the elliptical shape of the left ventricle. In order to apply the correct image motion constraints to the model, a set of "model tags" was created within the FE model. Model tags represent the material surfaces within the heart tissue which are tagged with magnetic saturation and which deform with the heart. 
The location and orientation of the tag planes at the time of their creation (ED) are determined by the tagging pulse sequence parameters. Each tag plane $P_{t}$ was described by a point on the plane $\mathbf{p}_{t}$ and a normal $\mathbf{n}_{t}$ to the plane. Similarly each image slice plane $P_{i}$ was described by a point $\mathbf{p}_{i}$ and normal $\mathbf{n}_{i}$. Each tag plane was associated with one or more image slice planes; let $T_{i}$ denote the set of tag planes associated with each image plane $P_{i}$. Model tags were found using a subdivision algorithm [15],[17]. Each element was subdivided into $\mathrm{N}$ subelements with subelement nodes equally spaced in $\xi$ space. The tag planes were then intersected with the subelement mesh assuming linear variation between subelement nodes. The result was a set of triangles whose vertices were given in element (material) coordinates. Fig. 1a shows a schematic of this procedure.

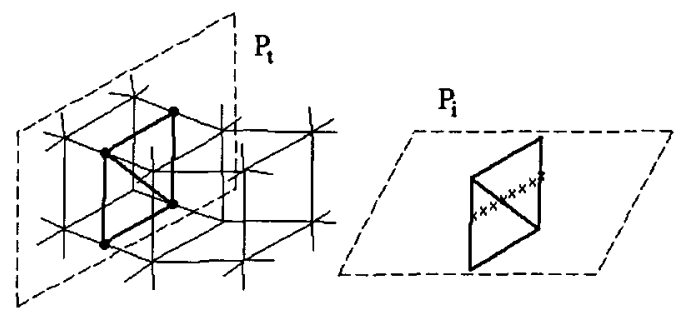

Fig. 1. Calculation of model tags (a, left) and model tag-image intersections (b, right). a) thin lines: model subelement boundaries; dashed lines: tag plane $P_{t}$; thick lines: model tag triangles. b) thick lines: model tag triangles; dashed lines: image plane $P_{i}$; $\mathbf{x}$ : MTI points.

\subsection{Model Deformation}

At any stage in the model fitting process, a collection of model tag - image intersection (MTI) points could be found by intersecting the model tags with the image planes. For each intersecting model tag triangle, the intersecting edges were interpolated to result in a set of MTI points spaced approximately $1-2 \mathrm{~mm}$ apart (Fig. 1b). Let $M(i, t)$ denote the set of MTI points associated with each $P_{t} \in T_{i}$. The model was deformed from frame to frame to minimize the following objective function:

$$
E(\mathbf{x})=S(\mathbf{x})+\sum_{P_{i}} \sum_{P_{t} \in T_{i}} \sum_{n \in M(i, t)} w_{n}\left[\mathbf{n}_{t} \cdot\left(\mathbf{x}\left(\xi_{n}\right)-\mathbf{x}_{n}\right]^{2}\right.
$$

where $S$ is a smoothing term, $\mathrm{x}\left(\xi_{n}\right)$ are MTI points associated with tag plane $P_{t}$ intersecting image $P_{i}$, and $\mathbf{x}_{n}$ are image stripe points associated with $\mathbf{x}\left(\xi_{n}\right)$. The $w_{n}$ are weights derived from the image intensity function and $\mathbf{n}_{t}$ are the normals to the original tagging plane $P_{t}$. The smoothing term measured the variation of 
the deformation from a prior geometry, as described previously [4]:

$$
S(\mathbf{x})=\int_{\Omega} \sum_{k}\left\|\frac{\partial \mathrm{F}}{\partial \xi_{k}}\right\|_{F}^{2} \partial \Omega
$$

where $\mathrm{F}$ is the deformation gradient tensor defined with respect to the rectangular Cartesian coordinate system. The smoothing weights were set small enough to have negligible effects in regions containing sufficient data points. In regions with few or no data points, the effect of this smoothing term is to reduce the variation of deformation across the model.

The error function eqn. (2) was minimized using an iterative nonlinear least squares procedure (see Appendix and [15]). In summary, each iteration consisted of the solution of eqn. (2) by linear least squares (keeping the $\xi_{n}$ constant). After each iteration, the MTI points $\mathbf{x}\left(\xi_{n}\right)$ were recalculated, along with their associated image stripe points $\mathbf{x}_{n}$. The dot product employed in eqn. (2) is a statement of the aperture problem: The image stripes provide information about where the material point is in the direction normal to the tag, whereas the position of the material point along the tag is unknown. This constraint is similar to that used previously in optical flow problems [18].

\subsection{Image Stripe Points}

Image stripe points were associated with each MTI point by searching the image in a small neighborhood for the most likely point on the image to which the MTI point should move. The likelihood function measured the probability that each pixel was located on the center of an image stripe. This was given by the output of stripe detection filters (one for each stripe orientation) convolved with the image. The filters had a Gaussian shape in the direction parallel to the stripe and a second derivative of a Gaussian in the direction normal to the stripe. The scale of the filter was tuned to the width of the tag stripes (in this paper all filters had $\sigma=1.5$ pixels). The search was carried out in a small neighborhood centered about each MTI point consisting of those pixels in the direction orthogonal to the image stripe and less than half a stripe spacing away from the MTI point. Rather than take the image point with the maximum filtered image value, the centroid of the neighbourhood (the average of all the points in the neighborhood weighted by their filtered image values) was used as the most likely stripe point. This was a more robust measure of the position of the stripe center than the maximum filtered value, and allowed the calculation of the image stripe center to subpixel resolution. Center-of-mass image constraints have previously been employed for active contour models and are more stable than gradient-based image constraints [19]. Finally, the weight $w_{n}$ for each neighbourhood was calculated as the maximum value of the filtered image in the neighborhood. Myocardial pixels with low weight are located between stripes (where the search neighborhood did not include a stripe center) and on stripes running in the other direction.

Previous studies have shown that image stripe orientations do not change substantially during the cardiac cycle (typically less than $20^{\circ}$ ). The search direction was therefore kept constant throughout the tracking process (orthogonal 
to the original stripes). This enabled the result of the search to be precalculated before the stripe tracking process. For each image, a displacement image was generated which stored the displacement in pixels from each pixel to the centroid of the filtered image in the search neighbourhood. To maintain subpixel resolution, the displacement was multiplied by a scaling factor before being stored as a 1 byte/pixel image. The weight for each pixel was similarly precalculated as an image. Fig. 2 shows an example of a short axis image at end-systole (ES), showing raw, filtered, displacement and weighting images.
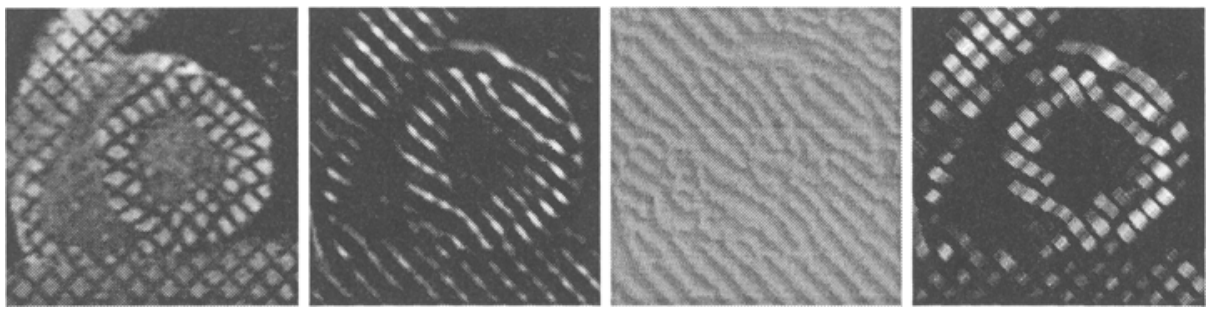

Fig. 2. Precalculation of image forces as images. From left: raw image; result of the stripe detection filter in one orientation; displacement image (lighter greylevels indicate displacement towards the top right, darker greylevels indicate displacement in the opposite direction); weight image (lighter greylevels indicate higher weighting)

\subsection{User Interaction}

It is not uncommon for model tags to move more than one stripe spacing between frames. In order to bring the model into approximate correspondence with the image stripes a fit to guide points was performed. This involved the minimization of eqn. (2) above with small number of guide points in place of the image stripe points. The smoothing term ensured a smooth interpolation between guide point displacements. To define a guide displacement the user clicked on a MTI point on the image and then clicked on a point to which the MTI point should move. The normal in the dot product of eqn. (2) was the tag normal associated with the MTI point. After these "guide iterations" the MTI points should be within range of the image stripe forces and the fit could proceed with "image iterations" as above. The user was also able to interact with the image iteration by selecting a group of MTI points and redefining their associated image stripe points.

\subsection{Image Acquisition}

In vivo clinical images of a normal volunteer were acquired with a Siemens Vision MR scanner using breath-hold segmented k-space imaging sequences. Eight parallel short axis slices were obtained orthogonal to the central axis of the LV spaced $11.5 \mathrm{~mm}$ apart, together with six long axis slices oriented $30^{\circ}$ apart about 
the LV central axis. Each slice was $8 \mathrm{~mm}$ thick and comprised 19 frames through the cardiac cycle. The image resolution ranged from 1.17 to $1.37 \mathrm{~mm}$ per pixel, depending on the field of view.

\subsection{Simulated Deformation}

Simulated images were also generated of a prescribed deformation, in order to determine whether errors are introduced in the calculation of model tags and MTI points or in the search for image stripe centroids. An initial regular ED geometry was constructed in prolate spheroidal coordinates (focal length of 42 $\mathrm{mm}$ ), with inner surface at $\lambda=0.60$ and outer surface at $\lambda=0.85$. The final ES geometry had an inner surface at $\lambda=0.40$ and outer surface at $\lambda=0.82$, with a longitudinal contraction of $15 \%$ in $\mu$ and uniform twist in $\theta$ from $24^{\circ}$ and $21^{\circ}$ at the endocardial and epicardial apex respectively to $-8^{\circ}$ and $-7^{\circ}$ at the endocardial and epicardial base respectively. The prolate model was converted to rectangular Cartesian coordinates and model tags ( $8 \mathrm{~mm}$ apart) were calculated for 8 short axis images spaced $10 \mathrm{~mm}$ apart and 6 long axis images spaced $30^{\circ}$ apart about the central axis. The images were then simulated by assigning each pixel within the inner and outer boundaries to a representative myocardial greylevel and each pixel within $1 \mathrm{~mm}$ of a MTI point to a representative stripe greylevel.

The simulated images were also analyzed using previously described stripe tracking and model fitting procedures [4]. Briefly, the stripes were tracked in each slice using an active contour model of the $2 \mathrm{D}$ tagging grid. The finite element model was then used to reconstruct the 3D displacements of material points by fitting the motion from ES to ED. Then the ED model was deformed to ES by fitting the reconstructed 3D displacements of the stripe data.

\section{Results}

\subsection{Initial Geometry}

In total 103 guide points were required for the ED geometry of the normal volunteer ( 53 for the endocardial surface and 50 for the epicardial surface). As there were 14 image slices this represents 3-4 points per surface per slice. The geometry in the third frame was determined first, then the guide points were moved to match the motion of the stripes back to the ED frame. The entire process required less than 10 minutes to complete, compared with approximately 30 minutes to manually define each boundary on each slice individually.

\subsection{Model Deformation}

Using the ideal tag plane positions and orientations derived from the imaging parameters, 182 model tags were found within the ED geometry. The model was then deformed to each frame by fitting the location of the MTI points to the 
image stripes. A small motion occurred between the tag creation and the time of the ED image, so this frame was also fitted in the same manner. The deformed ED geometry was then used as the prior (undeformed state) in the smoothing term for all subsequent frames. Fig. 3 shows three of the model tags (two from the short axis images and one from a long axis image) at tag creation and ES. Fig. 3
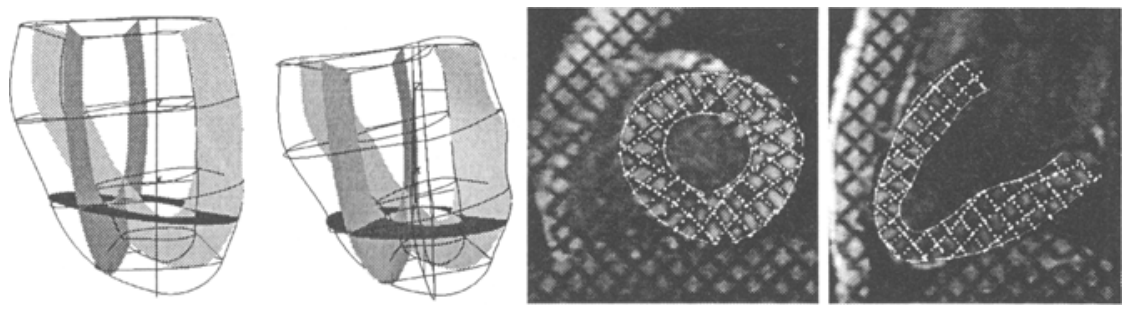

Fig. 3. a (left): Model tags at the time of creation (left) and at ES (right). The two lighter tags are from the short axis images, while the dark tag is from a long axis image. b (right): Short (left) and long (right) axis images at ES with MTI points overlayed.

shows short and long axis images at ES with MTI points overlayed. Typically, one guide iteration ( $\sim 30$ guide points) and two image iterations ( $\sim 4900$ points) were sufficient to achieve good correspondence between image stripes and MTI points.

The 3D tracking procedure took $\sim 5$ min per frame, representing a considerable time saving over the previous method [4] which required definition of the inner and outer boundaries and image stripe tracking for each frame followed by 3D model fitting ( $\sim 5$ min per frame). Each iteration (least squares fit) took $\sim 30 \mathrm{sec}$ to compute on a $180 \mathrm{Mhz}$ R5000 workstation, however the code has not been optimized for execution speed.

\subsection{Simulated Deformation}

The simulated ED geometry was deformed to fit the simulated image stripes in the same manner as above. Typically, 2 guide points per frame were required to bring the model into approximate correspondence. The root mean squared (RMS) errors in the displacement were $0.38 \mathrm{~mm}$ in $\mathrm{x}, 0.34 \mathrm{~mm}$ in $\mathrm{y}$ and 0.34 $\mathrm{mm}$ in $\mathrm{z}$ (the image pixel size was $1.17 \mathrm{~mm}$ ). RMS errors in strain were 0.022 in circumferential strain (true range -0.357 to 0.088 ), 0.021 in longitudinal strain (true range -0.350 to -0.117 ) and 0.187 for radial strain (true range 0.446 to $0.930)$.

These images were also analyzed using a previously described 2D stripe tracking algorithm and $3 \mathrm{D}$ finite element model fitting technique. The RMS errors of the resulting displacement were $0.29 \mathrm{~mm}$ in $\mathrm{x}, 0.38 \mathrm{~mm}$ in $\mathrm{y}$ and $0.38 \mathrm{~mm}$ in $\mathrm{z}$. Strain estimates had RMS errors of 0.023 in circumferential strain, 0.020 in longitudinal strain and 0.186 in radial strain. Thus the direct $3 \mathrm{D}$ tracking method 
resulted in similar errors to the 2D tracking/3D fitting method. The error was greatest in the radial (transmural) direction and was mainly due to the lack of tag resolution in this direction, especially at the apex where there was only one stripe orthogonal to the radial direction.

\section{Discussion}

This paper describes a method for directly reconstructing the shape and motion of the LV. Use of a 3D model to track the stripes implicitly constrains the motion in each slice to be compatible with its $3 \mathrm{D}$ neighbours. Thus, all stripes in all images contribute to the tracking process in a coherent $3 \mathrm{D}$ manner. User interaction is also constrained to act on the 3D model, thereby maintaining compatibility between displacements and reducing the amount of interaction to a minimum. Features of the method were: a) the use of very sparse guide points in combination with model smoothing to estimate the approximate geometry at ED and gross motion of the LV, b) the calculation of model tags and MTI points for use in the objective function eqn. (2) and c) precalculation of the image displacements, reducing the search for corresponding image stripe points to a simple look-up operation.

Park et al. [8],[9] developed a class of deformable model with parametric global deformations which varied locally to capture the heart motion. Rather than model the tag surfaces, tag planes were assumed to translate without bending in the through-plane direction from frame to frame. O'Donnell et al. [10],[11] described a deformable model with both global and local components of motion. Deformation parameters were fitted to a priori contour and stripe data. The 3D displacements of the stripe data were reconstructed by estimating the motion from each deformed frame back to the first frame (ED), as in [4]. Denney [14] described a stripe tracking procedure which did not require prior knowledge of the inner and outer contours of the heart. An active contour model was used to track stripes across an entire region of interest and a maximum a posteriori hypothesis test was used to segment myocardial tag stripes from background. Such contour-free stripe data could be used as input to model- free motion reconstruction methods such as the method developed by Denney and McVeigh [12], or that of Kerwin and Prince [20]. These methods do not require the prior formation of a geometric model; however regional analysis of material strains requires further processing.

\section{References}

1. Axel, L. and L. Dougherty. Heart Wall motion: Improved method of spatial modulation of magnetization for MR imaging. Radiology. 172: 349-350, 1989.

2. Zerhouni, E.A., D.M. Parish, W.J. Rogers, A. Yang and E.P. Shapiro. Human heart: Tagging with MR imaging - A method for noninvasive assessment of myocardial motion. Radiology. 169: 59-63, 1988. 
3. Young, A.A., L. Axel, L. Dougherty, D.K. Bogen and C.S. Parenteau. Validation of tagging with MRI to estimate material deformation. Radiology. 188: 101-108, 1993.

4. Young, A.A., D.L. Kraitchman, L. Dougherty and L. Axel. Tracking and finite element analysis of stripe deformation in magnetic resonance tagging. IEEE Trans Medical Imaging. 14: 413-421, 1995.

5. Palmon L.C., N. Reichek, S.B. Yeon, N.R. Clark, D. Brownson, E. Hoffman, and L. Axel. Intramural myocardial shortening in hypertensive left ventricular hypertrophy with normal pump function. Circulation, 89(1):122-131, 1994.

6. Young, A.A., C.M. Kramer, V.A. Ferrari, L. Axel and N. Reichek. Threedimensional left ventricular deformation in hypertrophic cardiomyopathy. Circulation. 90: 854-867, 1994.

7. O'Dell, W.G., C.C. Moore, W.C. Hunter, E.A. Zerhouni, and E.R. McVeigh. Threedimensional myocardial deformations: Calculation with displacement field fitting to tagged MR images. Radiology, 195(3):829-835, 1995.

8. Park, J., D. Metaxas, A.A. Young, and L. Axel. Deformable models with parameter functions for cardiac motion analysis from tagged MRI data. IEEE Trans. Medical Imaging, 15(3):278-289, 1996.

9. Park, J., D. Metaxas, and L. Axel. Analysis of left ventricular wall motion based on volumetric deformable models and MRI-SPAMM. Medical Image Analysis, 1(1):53$71,1996$.

10. O'Donnell, T., A. Gupta and T. Boult. The hybrid volumetric ventriculoid: New model for MR-SPAMM 3-D analysis. Proceedings of Computers in Cardiology, pp 5-8, Vienna, September 1995.

11. O'Donnell, T., T. Boult and A. Gupta. Global models with parametric offsets as applied to cardiac motion recovery. Proceedings of Computer Vision and Pattern Recognition, pp 293-299, San Francisco, June 1996.

12. Denney T.S. Jr. and E.R. McVeigh. Model-free reconstruction of three-dimensional myocardial strain from planar tagged MR images. J. Magnetic Resonance Imaging, 7:799-810. 1997.

13. Guttman, M.A., J.L. Prince and E.R. McVeigh. Tag and contour detection in tagged MR images of the left ventricle. IEEE Trans. Medical Imaging, 13(1): 7488, 1994.

14. Denney, T.S. Jr., Identification of myocardial tags in tagged MR images without prior knowledge of myocardial contours. XVth International Conference on Information Processing in Medical Imaging, pp 327-340, Poultney, Vermont, June 1997.

15. Young A.A., F.A. Fayad and L. Axel. Right ventricular midwall surface motion and deformation using magnetic resonance tagging. American Journal of Physiology: Heart and Circulatory Physiology. 271:H2677-H2688, 1996.

16. Terzopoulos, D. The computation of visible-surface representations. IEEE Trans. Pattern Analysis and Machine Intelligence. 10: 417-438, 1988.

17. Lorensen, W.E. and H.E. Cline. Marching cubes: A high resolution 3D surface construction algorithm. Computer Graphics. 21: 163-169, 1987.

18. Hildreth, E.C. Computations underlying the measurement of visual motion. Artificial Intelligence 23:309-354, 1984.

19. Davatzikos, C.A. and J.L. Prince. Convexity Analysis of Active Contour Problems. Proceedings of Computer Vision and Pattern Recognition, San Francisco, June 1996.

20. Kerwin, W.S. and J.L. Prince, J.L. Generating 3D cardiac material markers using tagged MRI. XVth International Conference on Information Processing in Medical Imaging. pp 313-326. Poultney, June 1997. 
21. Marquardt, D.W. An algorithm for least squares estimation of nonlinear parameters. J Soc Indust Appl Math. 11: 431-441, 1963.

\section{Appendix: Minimization Algorithm}

A Levenburg-Marquardt algorithm [21] was used to minimize eqn. (2), as done previously for RV surface models [15]. The objective function eqn. (2) can be written:

$$
E=\|\mathrm{Sq}\|^{2}+\|\mathrm{Jq}-\mathbf{p}\|^{2}
$$

where $\mathrm{S}$ is a matrix derived from the smoothing term, $\mathrm{J}$ is a matrix containing model basis functions evaluated at $\xi_{n}$ and weighted by the tag normals, $\mathbf{p}$ is a vector containing components of $\mathbf{x}_{n}$ weighted by the tag normals and $\mathbf{q}$ is $\mathbf{a}$ vector of model parameters. Note that $\mathbf{J}$ varies with $\mathbf{q}$, but $\mathbf{S}$ does not.

The Newton method minimizes $E$ by neglecting terms higher than second order in the Taylor series expansion, giving the following iteration:

$$
\mathrm{H}_{k}\left(\mathbf{q}_{k+1}-\mathbf{q}_{k}\right)=-\left.\frac{\partial E}{\partial \mathbf{q}}\right|_{k}
$$

where $H$ is the Hessian matrix of second derivatives of $E$. The right hand side of eqn. (5) has a component due to the fact that $\xi_{n}$ can change with q. However, the error function eqn. (2) measures the squared distance from each data point to the model position $\xi_{n}$ in the direction approximately perpendicular to the model tag surface. Since $\xi_{n}$ can only change within the model tag surface, the contribution to the first derivative of the error function due to changes in model position will be small. We therefore use the linear approximation:

$$
\frac{\partial E}{\partial \mathbf{q}}=\mathrm{S}^{T} \mathrm{~S} \mathbf{q}+\mathrm{J}^{T} \mathrm{Jq}-\mathrm{J}^{T} \mathbf{p}
$$

Replacing the Hessian $\mathrm{H}$ with the linear approximation $\mathrm{S}^{T} \mathrm{~S}+\mathrm{J}^{T} \mathrm{~J}$, and adding a term $\Lambda I(\Lambda>0)$ to avoid non-positive definite $\mathrm{H}$, gives the iteration:

$$
\left(\mathrm{S}^{T} \mathrm{~S}+\mathrm{J}_{k}^{T} \mathrm{~J}_{k}+\Lambda I\right) \mathbf{q}_{k}+1=\Lambda I+\mathrm{J}_{k}^{T} \mathbf{p}
$$

If $\lambda$ is large the step becomes small and in the direction of steepest descent; if $\lambda$ is small the update becomes a full Gauss-Newton step. In practice, all the iterations were performed with $\lambda=0$, equivalent to solving the linear problem that arises if the $\xi_{n}$ are assumed to be constant over the step. 